\title{
Cetoacidosis diabética en niños: un reto para el clínico
}

\author{
Diabetic ketoacidosis in children: A challenge for the clinician
}

Si de por sí la condición de cetoacidosis diabética consiste en una estado clínico de gravedad, lo es aún más en los niños, en los que reconocer esta entidad se vuelve todo un reto para el clínico desde su identificación hasta el tratamiento, el cual no sólo consiste en lograr un estado metabólico adecuado, sino en evitar las complicaciones que derivan de ésta.

La diabetes mellitus afecta a gran parte de la población mundial. Según datos de los Centros para el Control y la Prevención de Enfermedades (CDC), para el año 2010, alrededor de 215.000 americanos menores de 20 años tenían diabetes [1]. La cetoacidosis es la primera manifestación de la enfermedad hasta en un tercio de los pacientes con diabetes mellitus tipo 1 ; lo mismo que ocurre para los pacientes con diabetes mellitus tipo 2 en un $5 \%$ al $25 \%$ [2].

La cetoacidosis diabética tiene una mortalidad menor del $1 \%$; sin embargo, es la primera causa de mortalidad y morbilidad en niños relacionada con la diabetes mellitus [3], con una incidencia de déficit neurológico secundario a edema cerebral mayor del $26 \%$, incluso con alteración de la memoria por la condición de cetoacidosis diabética per se aún sin edema cerebral $[4,5]$.

Aunque la triada clínica clásica de la cetoacidosis diabética consiste en poliuria, polidipsia y pérdida de peso, se debe sospechar esta entidad en todo paciente que curse con taquipnea y alteración en el nivel de consciencia, ya que la clínica en la población pediátrica especialmente en los lactantes puede ser muy atípica [6].

La causa metabólica de esta entidad parte del déficit absoluto o relativo de la insulina, lo que genera un incremento significativo de las hormonas contrarreguladoras con hiperglucemia secundaria $[6,7]$, la cual se perpetúa aún más por la deshidratación. Es fundamental conocer las bases fisiopatológicas de esta crisis hiperglucémica aguda para dirigir el tratamiento, donde se parte desde un estado de acidosis metabólica y cetosis sumado a deshidratación, lo que implica brindar una adecuada hidratación e insulinoterapia dirigida, no sólo a lograr una meta glucémica, sino con el objetivo de inhibir la cetogénesis.

Se debe hacer una búsqueda clínica activa del posible factor desencadenante de la crisis hiperglucémica, el cual hasta en un 30\% de los casos puede ser debida a una infección asociada [8]; además, considerar diagnósticos diferenciales de acuerdo a la clínica de cada paciente, en los cuales se debe tener presente otras causas de enuresis secundaria, de dolor abdominal, de taquipnea, de alteraciones en el nivel de consciencia y de posibles intoxicaciones por salicilatos, metanol, etilenglicol y paraldehído [9].

Una vez se alivia esta condición clínica se debe resolver la causa precipitante y mantener un estado euglucémico con una adecuada dosificación de insulina, la cual 
dependerá del estado puberal y el contexto clínico de sensibilidad/resistencia a la insulina de cada niño, con base en un esquema basal-bolo en el cual la mitad de la dosis total diaria de insulina se administra con insulina de larga acción (basal) y la mitad restante se distribuye de forma uniforme en cada comida (bolo) y se titula la dosis de acuerdo a la respuesta glucémica y por la identificación de patrones de sensibilidad/resistencia a lo largo del día.

Se deben tener muy presente las complicaciones dadas tanto por la condición de acidosis metabólica como por el tratamiento instaurado, las cuales pueden ir desde sobrecarga hídrica hasta trastornos electrolíticos, principalmente hipokaliemia, como es el caso del edema cerebral con posterior compromiso neurológico.

Por todo lo anterior, se considera que es fundamental para el clínico identificar de forma temprana esta condición en los niños y tener a la cetoacidosis diabética siempre presente entre sus diagnósticos diferenciales, sobre todo en los lactantes donde la clínica puede ser atípica, lo que retarda el diagnóstico y con ello incrementa la morbilidad y mortalidad en este grupo poblacional; además, dirigir un tratamiento basado en una hidratación adecuada, resolución de la cetosis, tratar el factor desencadenante y evitar complicaciones.

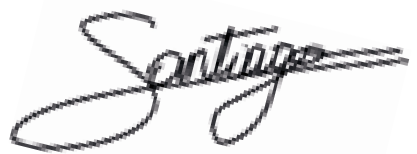

\section{Santiago Saldarriaga Betancur, MD.}

Médico, Grupo de investigación IDEAS (Innovación, Desarrollo y Avances en Endocrinología)/ Clínica Integral de Diabetes (CLID)

Medellín, Colombia, diciembre 2016

\section{Bibliografía}

1. Centers for Disease Control and Prevention. National diabetes fact sheet: national estimates and general information on diabetes and prediabetes in the United States, 2011. Atlanta, Estados Unidos: U.S. Department of Health and Human Services, Centers for Disease Control and Prevention. 2011. Disponible: https://www.cdc.gov/ diabetes/pubs/pdf/ndfs_2011.pdf.

2. Klingensmith GJ, Tamborlane WV, Wood J, Haller MJ, Silverstein J, Cengiz E, et al. Diabetic ketoacidosis at diabetes onset: still an all too common threat in youth. J Pediatr 2013; 162: 330-334 e331.

3. Edge JA, Ford-Adams ME, Dunger DB. Causes of death in children with insulin dependent diabetes 1990-96. Arch Dis Child 1999; 81: 318-323.

4. Edge JA, Hawkins MM, Winter DL, Dunger DB. The risk and outcome of cerebral oedema developing during diabetic ketoacidosis. Arch Dis Child 2001; 85: 16-22.
5. Ghetti S, Lee JK, Sims CE, Demaster DM, Glaser NS. Diabetic ketoacidosis and memory dysfunction in children with type 1 diabetes. J Pediatr 2010; 156: 109-114.

6. Kitabchi AE, Umpierrez GE, Murphy MB, Barrett EJ, Kreisberg RA, Malone JI, et al. Hyperglycemic crises in diabetes. Diabetes Care 2004; 27 Suppl 1: S94-102.

7. Coll-Barrios $\mathbf{M}$, Durán-Ventura $\mathbf{P}$, Mejía L Enfoque práctico de la cetoacidosis diabética en pediatría. CCAP 2008; 7: 1-9.

8. Kitabchi AE, Umpierrez GE, Murphy MB, Barrett EJ, Kreisberg RA, Malone JI, et al. Management of hyperglycemic crises in patients with diabetes. Diabetes Care 2001; 24: 131-153.

9. Wolfsdorf JI, Allgrove J, Craig ME, Edge J, Glaser N, Jain V, et al. ISPAD Clinical Practice Consensus Guidelines 2014. Diabetic ketoacidosis and hyperglycemic hyperosmolar state. Pediatr Diabetes 2014; 15 Suppl 20: 154-179. 